\title{
REVIEW
}

\section{Citrus Breeding 2.0: A Novel Approach Integrating Deciphered Parentage and Genomics-assisted Selection}

\author{
Tokurou SHIMIZU* \\ Division of Citrus Research, Institute of Fruit Tree and Tea Science, NARO (Shimizu, Shizuoka \\ 424-0292, Japan)
}

\begin{abstract}
Current citrus breeding programs have three objectives: developing diverse varieties that satisfy assorted commercial needs; improving overall fruit quality; and releasing varieties quickly. However, these objectives may conflict with each other owing to the limited available orchard space, and conventional breeding systems have not progressed rapidly enough to resolve this problem. "Citrus Breeding 2.0" is a novel approach to overcome the trilemma of citrus breeding. "Citrus Breeding 2.0 " will increase diversity by referring to parentage, improve the overall fruit quality through composite selection, and minimize the breeding time through genomics-assisted selection. Extended applications of "Citrus Breeding 2.0" are also possible for purposes of rebreeding or improving an existing variety.

Discipline: Plant breeding

Additional key words: genome-wide association study (GWAS), genome-wide genotyping, genomic selection, parentage estimation, pedigree analysis
\end{abstract}

\section{Introduction}

To date, the crossbreeding program of the Institute of Fruit Tree and Tea Science, NARO (NIFTS), has released more than 40 citrus varieties. These varieties were intended for the domestic market, but developing new varieties for foreign markets is also necessary owing to the declining population of Japan. Export-orientated citrus breeding requires improving the overall quality of fruit to meet the demands of each destination country. Developing a new citrus variety in a minimum timespan is necessary to make breeding for international consumption viable. Additionally, developing a variety resistant to environmental variation and the emergence of new diseases and pests caused by rapid climate change is urgently required. Consequently, the citrus breeding program must achieve three simultaneous objectives: developing diverse varieties that satisfy assorted commercial needs, improving overall fruit quality, and releasing new varieties as quickly as possible. However, these three goals present a trilemma that is hard to resolve using conventional breeding. Recently, the parentages of many indigenous citrus varieties were identified, and it was revealed that they play pivotal roles in several varieties. In addition, new genomics-assisted selection techniques avoid the constraints of the usual quantitative trait loci (QTL) analyses. Here, we introduce a new breeding strategy in citrus, "Citrus Breeding 2.0," for resolving the trilemma by combining the revealed parentages and new genomicsassisted selection technologies. Furthermore, extended applications of "Citrus Breeding 2.0" for rebreeding or for improving an existing variety are discussed.

\section{Overview of citrus breeding at the NIFTS}

\section{Features of citrus breeding}

The citrus breeding program of the NIFTS obtains more than 5,000 seedlings from various crosses annually. A promising scion is selected after an extended stepwise evaluation of $\sim 20$ traits, but it sometimes fails to pass the required quality threshold. The average length for the first blooming is 6-7 years, and the following field evaluations require an extended period. A candidate scion is also subjected to strain adaptability testing. At the NIFTS, it 
took an average of 22.8 years (11-38 years) for 36 diploid hybrid varieties that were crossed from 1946 to 1998 to go from pollination to the official naming registration or variety registration ('Tsunonozomi' and 'Aurastar'). The selected scions were propagated by grafting and then supplied to nursery growers.

\section{Constraints on current citrus breeding}

The genus Citrus consists of more than 150 accessions that show large variations. The genomes of many indigenous varieties are highly heterozygous (Shimizu et al. 2016a), which contributes to enlarge the variation, even in small populations. However, the large tree size restricts the number of trees that can be planted per acre. Due to the fact that most fruit traits are quantitative and that citrus trees set fruit annually, repeating an evaluation over several years is mandatory to increase the accuracy of the evaluation. These constraints extend the time for trait evaluation required by a QTL analysis and make it difficult to verify the effects of detected QTLs in other crosses.

\section{Trilemma in citrus breeding}

\section{Increasing fruit trait variation}

A few elite citrus varieties, such as Satsuma, sweet orange, hassaku and Kiyomi, have frequently been used as breeding parents (Table 1). In particular, Kiyomi is used as a parent in 16 varieties because of its sweet orange-like aroma, monoembryonic nature, and improved peelability compared with sweet orange. As a result, considerable numbers of varieties developed from Kiyomi tend to show similar characteristics. Other citrus varieties, such as lemon, hyuganatsu, Kishu, and natsudaidai, have been

Table 1. Frequencies of varieties used in the citrus breeding program at the NIFTS

\begin{tabular}{|c|c|c|c|}
\hline Parental varieties & $\begin{array}{l}\text { As a parent } \\
\text { of varieties }\end{array}$ & $\begin{array}{l}\text { As a parent } \\
\text { of selections }\end{array}$ & Scientific name \\
\hline Satsuma & 7 & 4 & C. unshiu Marc. \\
\hline Ponkan & 6 & 0 & C. reticulata Blanco \\
\hline Hassaku & 4 & 1 & C. hassaku hort. ex Tanaka \\
\hline Hirado buntan & 4 & 0 & C. maxima Merr. \\
\hline Sweet orange & 3 & 4 & C. sinensis (L.) Osbeck \\
\hline Clementine & 2 & 0 & C. clementina hort. ex Tanaka \\
\hline Mato buntan & 2 & 0 & C. maxima Merr. \\
\hline Tanikawa buntan & 1 & 0 & C. maxima Merr. \\
\hline Banpeiyu & 1 & 0 & C. maxima Merr. \\
\hline Kishu & 1 & 0 & C. kinokuni hort. ex Tanaka \\
\hline Natsudaidai & 1 & 0 & C. natsudaidai Hayata \\
\hline Lemon & 1 & 0 & C. limon (L.) Burm. f. \\
\hline Hyuganatsu & 1 & 0 & C. tamurana hort. ex Tanaka \\
\hline Iyo & 0 & 1 & C. iyo hort. ex Tanaka \\
\hline Trifoliate orange & 0 & 1 & Poncirus trifoliata L. \\
\hline Kiyomi & 10 & 6 & Satsuma $\times$ Sweet orange \\
\hline Encore & 6 & 3 & King $\times$ Willowleaf mandarin \\
\hline Harumi & 2 & 0 & Kiyomi $\times$ Ponkan \\
\hline Murcott & 2 & 0 & C. spp (hybrid?) \\
\hline Sweet spring & 0 & 3 & Satsuma $\times$ Hassaku \\
\hline Yoko & 1 & 0 & Kiyomi $\times$ Ponkan \\
\hline Tsunonozomi & 1 & 0 & Kiyomi $\times$ Encore \\
\hline Awa orange & 1 & 0 & Hyuganatsu $\times$ Sweet orange \\
\hline Kara & 1 & 0 & Satsuma $\times$ King mandarin \\
\hline Seminole & 1 & 0 & Grapefruit $\times$ Dancy \\
\hline Minneola & 1 & 0 & Grapefruit $\times$ Dancy \\
\hline Page & 1 & 0 & Mineola $\times$ Clementine \\
\hline Wilking & 1 & 0 & King $\times$ Willowleaf mandarin \\
\hline Osceola & 0 & 1 & Clementine $\times$ Orland \\
\hline Hybrid selection & 12 & - & \\
\hline
\end{tabular}

The list was compiled from the hybrid varieties and selections available at http://www.naro.affrc.go.jp/ nifts/kih/files/nifts_hinshu_ichiran.pdf. 
used as parents to incorporate a novel characteristic into the new selection. These minor varieties are superior in aroma, acidity, or taste compared with other frequently used varieties, but their other traits are often insufficient to be used as a parent for breeding. Among these traits, fruit size strictly determines candidate selection, even though many mandarin varieties set small-sized fruits. Other inferior traits of those minor varieties such as higher acidity, less peelability, lower sugar content (Brix), or an undesirable taste or aroma, often hamper them in being used as a breeding parent. A method of segregating desirable novel traits in minor varieties from undesired traits will allow their use and increase diversity in new scions. Selecting a potential variety for this purpose is another essential point to consider.

\section{Improving overall fruit quality}

Marker-assisted selection (MAS), for which a DNA marker linked to a quantitative trait is used, is a valid method for selecting a candidate. Increasing the number of selectable traits by MAS contributes to the improvement of overall fruit quality. Owing to the long juvenile period of citrus, performing MAS at the young seedling stage will result in a drastic decrease in the cost of breeding (Shimizu 2018). The current citrus breeding program at the NIFTS provides $\sim 1,000$ seedlings annually for grafting at the orchard, which represents the actual scale of breeding. Most fruit-related characteristics are quantitative traits, and QTL analyses have been conducted to identify loci that influence a particular trait. QTL analyses strongly rely on the quality of data with regards to the trait of interest; however, the environment often affects specific traits. Annual evaluations of the same genotype, identification of other QTLs from other crosses, and verification of the availability of DNA markers linked to a QTL in various crosses are essential for increasing the availability of DNA markers for selection. However, these approaches often conflict with each other because of limited available orchard space. Consequently, a new approach is necessary that extends MAS for various traits.

\section{Demands for fast breeding}

Hybrid varieties with desired qualities have been developed after several generations. Stepwise selection is the usual process in many breeding programs, but it requires an extended period in citrus breeding. Using minor varieties to introduce novel traits is an attractive approach. However, it often requires excluding undesirable traits through consecutive crossing. For example, many citrus varieties are susceptible to a severe viral disease caused by citrus tristeza virus (CTV), which is common in Japan, and introducing CTV resistance to new hybrid varieties was requested to stabilize production. Among Rutaceae plants, trifoliate orange (Poncirus trifoliate) has strong and stable CTV resistance. It is a dominant trait controlled by a single gene (Gmitter et al. 1996). Ohta et al. (2011) developed DNA markers for its selection. However, the fruit of trifoliate orange is not edible, so it has been backcrossed with edible varieties. The CTVresistant hybrid varieties 'Aurastar', 'Nou No.7', and 'Nou No.8' were selected from a cross of 'H.FD-1', which was selected from hassaku and trifoliate orange 'Hiryu' cross. These three varieties are the F2 of trifoliate orange, but their fruit quality is not yet suitable for commercial production, and further backcrossing with edible varieties is required.

Fruit size is an important trait to consider through the selection. It is a typical quantitative trait that shows continuous distribution. Combrink et al. (2013) reported normal distributions of the height and width of fruit in six Kiyomi offspring populations crossed with different varieties. In their study, the medians for fruit size and shape were smaller than those of Kiyomi, but were close to those of varieties having smaller fruits. Combrink et al. (2015) also reported similar results for rind color. As many indigenous varieties set small-sized fruits compared with elite varieties, using these indigenous varieties for hybridization tends to result in smaller fruits on average, which decreases the possibility of obtaining candidates. This is a drawback when using non-elite indigenous varieties. Selecting multiple candidates from a single cross is mandatory for developing a wide range of variety in minimum time period, but the extended generation period conflicts with the time required for breeding, thus producing the trilemma of citrus breeding.

\section{Features and benefits of "Citrus Breeding 2.0"}

"Citrus Breeding 2.0" is an approach to increase the diversity of traits, improve the overall fruit quality by the composite selection of multiple traits, and minimize the time for selection through use of genomics-assisted selection.

\section{Use of citrus genealogy to increase the diversity of fruit traits}

Shimizu et al. (2016b) investigated the parentage of 22 indigenous varieties and identified their seed or pollen parent. They also inferred the parentage of 45 varieties. A few citrus varieties, such as tachibana, kunenbo, yuzu, koji, Kishu, and kaikoukan, played pivotal roles in the development of diverse citrus varieties. Among the varieties, tachibana, which had been recognized as having a single genotype, was determined to have three 
different genotypes. Additionally, four varieties (hanayu, girimikan, hyuganatsu, and oogonkan) were the offspring of tachibana. The fruit traits of the four varieties were entirely different in fruit size, rind thickness, peelability, and color of rind or flesh from tachibana. Tachibana has not been used as a breeding parent because it produces small, seedy, acidic, and puffy rind fruit. However, the revealed parentage strongly suggests its potential as a parent for developing scions with unique features. Kunenbo was also identified as the parent of 16 varieties, including Satsuma, Yatsushiro, kabosu, hassaku, and kaikoukan, which demonstrated the importance of the development of modern citrus varieties. Additionally, Shimizu et al. confirmed using whole-genome sequencing analysis that Kishu is a parent of kunenbo (Shimizu et al. 2017). The fruit size of kunenbo is large enough as a parent, but it has an unpleasant smell and is hard to peel. Interestingly, most of its offspring do not inherit the smell or hard skin traits. This suggests the possibility of eliminating those undesirable traits by segregating them from the desirable traits.

\section{Composite selection of overall fruit quality}

Genomic selection (GS) is a promising approach to predict a phenotypic value based on genome-wide genotype data and a prediction model (Iwata et al. 2016). In citrus, Minamikawa et al. (2017) developed GS models based on 111 citrus varieties and 676 individuals from 35 full-sibling families using both phenotypic data for 17 traits and genotypes of 1,841 single nucleotide polymorphism markers. They then demonstrated the ability of GS to predict those traits with high accuracy (Minamikawa et al. 2017). The prediction accuracy varied depending on the family and precision of the trait data. However, the developed GS model predicted with accuracy the fruit weight, even when the applied model was developed using insufficient data from large-sized varieties. This suggested the potential of GS for trait prediction even if the applied data set was small. The application of GS enables the selection of various fruit traits that was hard to achieve by conventional QTL analyses. Furthermore, it has the advantage of being able to select various traits using genotype datasets by changing the prediction model of GS. Minamikawa et al. (2017) also identified genomic regions that significantly correlated with 11 traits in a genome-wide association study (GWAS). The wide availability of QTLs detected by GWAS for a broad range of varieties benefits QTL analyses. Continuous identification of DNA markers by GWAS and studies verifying their performance are anticipated. GWAS estimates the effect of a particular genomic region that is associated with a trait, and it will help develop DNA markers for selection (Oraguzie et al. 2007). By contrast, GS estimates the contribution of many loci that have very faint effects on a trait from genomewide genotype datasets. Consequently, using QTL-linked markers developed by GWAS in combination with GS would be optimum for selecting multiple traits in various crosses and will contribute to improving the overall fruit quality.

\section{Fast breeding}

Estimating the best combination for a cross by referring to the parentages of known varieties and by selecting a promising scion that has novel traits from a single cross are keys to minimizing the breeding period. The probability of obtaining candidate scions depends on the number of seedlings available for MAS. The number of seedlings obtained from various crosses is greater than the number of seedlings that will be grafted at the orchard, and most of the seedlings will be discarded without evaluation. Therefore, conducting a composite selection on all seedlings before grafting and also using the selected seedlings for grafting will increase the probability of obtaining more candidates from the same acreage per generation. Although MAS does not alter the time for each generation, it will minimize the total breeding period by selecting a promising seedling from a single cross and will eliminate consecutive crosses. However, obtaining a genome-wide genotype dataset for GS is costly and determines the total cost. Applying a two-step selection that consists of MAS for all seedlings using small numbers of DNA markers that provide the primary candidates for subsequent evaluation by GS will minimize the cost. The choice of traits for the selection will also influence the cost and breeding efficiency. For example, Brix content is an essential selection trait, but a GWAS detected no obvious associated QTLs in citrus. However, a GS model predicted the Brix content with a moderate regression coefficient. The GWAS analysis have detected QTLs associated with several traits, such as FruH, ColorP, and ColorF (Minamikawa et al. 2017), and these will be useful for MAS performed in the initial selection. Applying known DNA markers during the initial selection will also be valuable.

\section{Applications of "Citrus Breeding 2.0"}

"Citrus breeding 2.0" will also be useful for the rebreeding of an existing variety or altering a particular trait in an existing variety. "Mimic" breeding reproduces an existing variety by hybridization. It selects a seedling that has a genome composition that resembles that of a particular known variety from the cross of the parents 
of known varieties. The citrus genome consists of nine chromosomes, and the probability of obtaining a particular chromosomal combination from the parental genome is $1 / 262,144$. The actual chance of obtaining the desired genome will be lower because of recombination, but selecting a seedling that has a genome composition that resembles that of the existing variety should produce a variety that is similar to the original. Another application, "complemental breeding," alters a specific trait of a variety by combining composite selection and "mimic" breeding. In citrus, novel mutants for particular traits have been identified from sports or nucellar seedlings. The complementary breeding initially selects individuals for the trait to be changed from the same cross as the target variety. The "mimic" breeding procedure will then select a candidate individual that has a genome that resembles that of the target variety. These two applications will fill the wide gap between crossbreeding and mutation breeding.

\section{Conclusion}

"Citrus Breeding 2.0" will increase trait diversity by referring to parentages, improving the overall fruit quality through composite selection, and minimizing the period for breeding, thereby avoiding the citrus breeding trilemma.

\section{References}

Combrink, N. K. et al. (2015) Genotypic variation of rind colour in citrus tangor Kiyomi families. Acta Hortic., 1065,
439-448. doi:10.17660/ActaHortic.2015.1065.54.

Combrink, N. K. et al. (2013) Variation of fruit size and shape in Kiyomi tangor families. Sci. Hortic. (Amsterdam), 162, 357-364. doi:10.1016/j.scienta.2013.08.010.

Gmitter, F. G. et al. (1996) A localized linkage map of the citrus tristeza virus resistance gene region. Theor. Appl. Genet., 92, 688-695. doi:10.1007/BF00226090.

Iwata, H. et al. (2016) Genomics-assisted breeding in fruit trees. Breed. Sci., 66, 100-115. doi:10.1270/jsbbs.66.100.

Minamikawa, M. F. et al. (2017) Genome-wide association study and genomic prediction in citrus: Potential of genomics-assisted breeding for fruit quality traits. Sci. Rep., 7, 4721. doi:10.1038/s41598-017-05100-x.

Ohta, S. et al. (2011) PCR primers for marker assisted backcrossing to introduce a CTV resistance gene from Poncirus trifoliata (L.) Raf. into Citrus. J. Japanese Soc. Hortic. Sci., 80, 295-307. doi:10.2503/jjshs1.80.295.

Oraguzie, N. C. et al. (2007) Association Mapping in Plants. New York, NY: Springer New York doi:10.1007/978-0-38736011-9.

Shimizu, T. (2019) Genomic breeding. In The Genus Citrus, eds. M. Talon, F. Gmitter, and M. Caruso (Elsevier), in press.

Shimizu, T. et al. (2016a) A genomic approach to selecting robust and versatile SNP sets from next-generation sequencing data for genome-wide association study in citrus cultivars. Acta Hortic., 1135, 23-32. doi:10.17660/ ActaHortic.2016.1135.4.

Shimizu, T. et al. (2016b) Hybrid origins of citrus varieties inferred from DNA marker analysis of nuclear and organelle genomes. PLoS One, 11, e0166969. doi:10.1371/ journal.pone.0166969.

Shimizu, T. et al. (2017) Draft sequencing of the heterozygous diploid genome of Satsuma (Citrus unshiu Marc.) using a hybrid assembly approach. Front. Genet., 8, 180. doi:10.3389/fgene.2017.00180. 